\title{
Study of nuclear fragmentation at MPD/NICA
}

\author{
M.B. Golubeva ${ }^{1, \star}$, A.P. Ivashkin ${ }^{1, \star \star}$, and A.B. Kurepin ${ }^{1, \star \star \star}$ \\ ${ }^{1}$ Institute for Nuclear Research RAS, Moscow, Russia
}

\begin{abstract}
Due to the much lower beam energy of NICA compared to the RHIC and LHC hadron colliders and the fixed target experiments at SPS the role and performance of the forward detectors of NICA are quite different. The Neutron Zero Degree Calorimeter could be used for the measurement and monitoring of luminosity, however with lower efficiency of neutron detection produced in ultra-peripheral collisions. The use of Forward Hadron Calorimeter for the determination of centrality is impossible by simply counting the number of spectators because of the ambiguity of the impact parameter dependence. This ambiguity could be removed if the angular distribution of the spectators will be taken into account. It is shown by the simulation with LAQGSM model that the forward multiplicity detector like V0 of ALICE could not be used for the determination of centrality. However it could provide the valuable information on the nuclear fragmentation of heavy ions.
\end{abstract}

Collider NICA will be the first collider working at much lower energy than RHIC and LHC. This fact should be taken into account by construction of the detectors systems of NICA. The barrel detectors like TPC, TOF, TRD and ECAL could be very similar due to the central geometry. But the performance of forward detectors is different because of the important contribution of nuclear fragments in the yield of produced particles in the collision. Therefore the specific role of forward detectors in the MPD/NICA project should be considered. From the experience of ALICE at LHC and STAR and PHENIX at RHIC it could be seen that forward detectors in the nucleus - nucleus collisions are mostly used for trigger and zero time formation and for centrality and reaction plane determination.

The most familiar method for the classification of the events by centrality is the use of the multiplicity forward detector, like V0 of ALICE [1]. The multiplicity measured by V0 detector is matched to the centrality classes corresponding to the gaps in percents of the impact parameter of collision [2]. The multiplicity distribution is rather smooth except of very peripheral events, which are accounted for by electromagnetic interaction with larger cross section.

Another method of centrality determination, used mostly for the experiments with the fixed target, is the measurements of the amplitude of the Zero Degree Calorimeter (ZDC), which is proportional to the number of the spectators emitted very close to the zero angle. The same method for collider is used with the Neutron ZDC [3] since charged fragments are deflected by the magnets in the beam line. For MPD/NICA the NZDC could be used for the measurement and monitoring of luminosity, however with lower efficiency of neutron detection produced in ultra-peripheral collisions [4]. The

\footnotetext{
^e-mail: marina@inr.ru

$\star \star$ e-mail: ivashkin@inr.ru

$\star \star \star$ e-mail: kurepin@inr.ru
} 
Forward Hadron Calorimeter (FHCAL), proposed for MPD/NICA, could not be used for determination of centrality by simply counting the number of spectators because of the ambiguity of the impact parameter dependence [5]. This ambiguity could be removed by the measurement of the asymmetry of signal amplitudes between the outer and inner modules of the calorimeter [6]. In this article the possibility to determine the centrality with the forward multiplicity detector (FMD) for MPD/NICA is investigated. This scintillator detector is segmented in several rings in the radial direction and divided in several sectors in the azimuthal direction with internal diameter $10 \mathrm{~cm}$ and external diameter 130 $\mathrm{cm}$. The thickness of scintillator is $2 \mathrm{~cm}$.
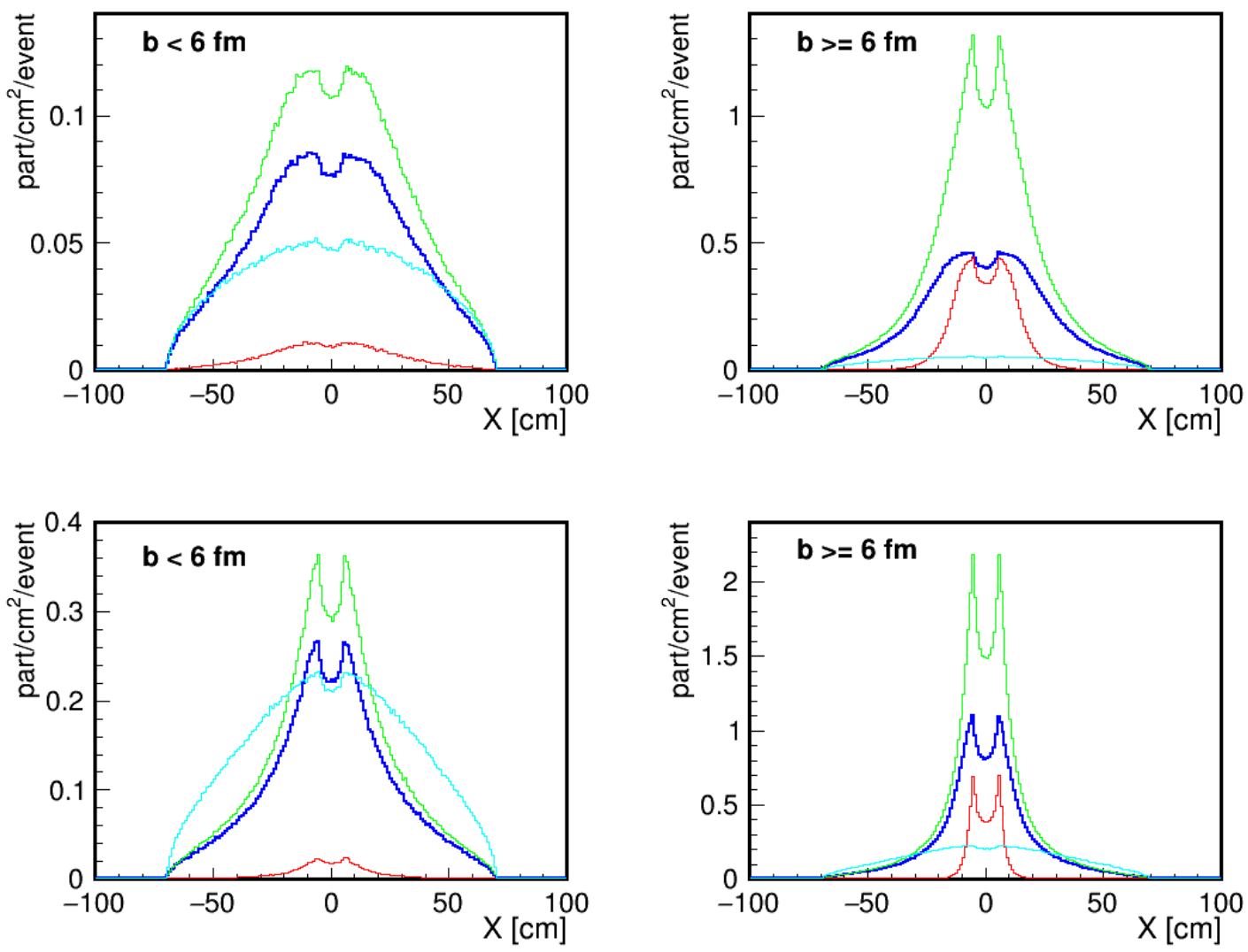

Figure 1. Horizontal distributions of protons (blue), neutrons (green), pions (light-blue) and fragments (red) produced in Au-Au collisions at $\sqrt{s}=5 \mathrm{GeV}$ (top) and $\sqrt{s}=11 \mathrm{GeV}$ (bottom) and impact parameters less than $6 \mathrm{fm}$ (left) and larger than $6 \mathrm{fm}$ (right).

For the simulation of Au-Au collisions the LAQGSM model [7] was used for the center of mass energies 5 and $11 \mathrm{GeV}$. In figure 1 the linear transverse spatial distributions are shown for protons, neutrons, pions and fragments $(\mathrm{A}>1)$ for collisions with the impact parameter less $6 \mathrm{fm}$ and larger 6 $\mathrm{fm}$. The minimum at the centre is the result of the beam hole. 

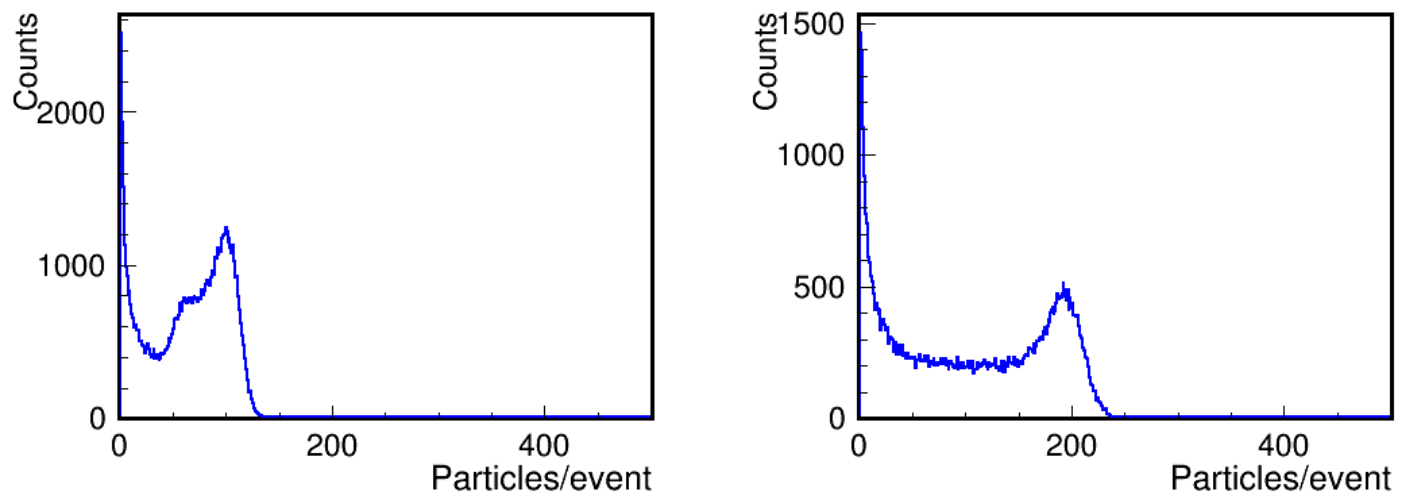

Figure 2. Charged particle multiplicity distributions at $\sqrt{s}=5 \mathrm{GeV}$ (left) and $\sqrt{s}=11 \mathrm{GeV}$ (right).
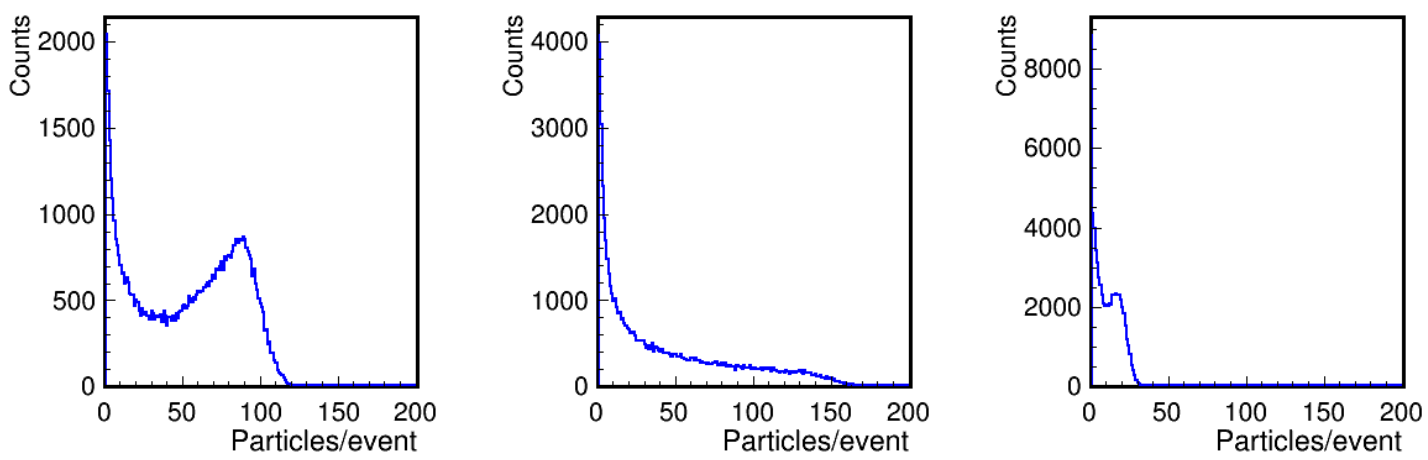

Figure 3. Multiplicity distributions of protons (left), pions (middle) and fragments (right) produced in $\mathrm{Au}-\mathrm{Au}$ collisions at $\sqrt{s}=11 \mathrm{GeV}$.

Calculated multiplicity distributions are shown in figure 2. The form of these distributions is different from that at high energy of ALICE. There are two maxima: one at small multiplicity and the second close to the limit of the number of particles per event.

It is seen from the calculations of multiplicity distributions made separately for protons, pions and fragments (figure 3) that the maximum at high multiplicity is due to protons, which could be considered as spectators from Au-Au collisions, arising at medium impact parameter. The same result was obtained for neutrons. This fact could change the common picture of the centrality determination in nucleus-nucleus collisions at intermediate energies. Because of Fermi motion of particle spectators they are deflected from the beam line and hit the surface of the FMD detector. However the emission of proton spectators is not uniform in the dependence on the impact parameter.

In the case of super-high energy of LHC in ALICE installation the spectators with high momenta do not hit the surface of $\mathrm{V} 0$ detector but pass inside the beam pipe. 
The mechanism of nuclear fragmentation in nucleus - nucleus collisions cannot be explained by simple geometrical picture. The study of nuclear fragmentation could be done at MPD/NICA with FHCAL, NZDC and possible FMD detectors in order to check different models of fragment production like Fermi break-up, preequilibrium emission and coalescence of produced particles. However for this purpose the FMD detector should be able to identify different fragments. This could be made with high granularity of FMD, energy loss and time-of-flight measurements. New information on fragment production will provide valuable information not only from scientific point of view, but also for many applications.

In conclusion we can note that the determination of the centrality of the collision in the case of relatively low beam energy of the NICA is not a simple problem. The spectators produced with sufficient transverse Fermi momentum are only partly hitting the detectors placed around the beam pipe, the heavier fragments are passing into the beam hole. Therefore the joint analysis of FHCAL, possible FMD and information from TPC and Neutron Zero Degree Calorimeter should be taken into account.

\section{References}

[1] The ALICE Collaboration, JINST 8, P10016 (2013)

[2] The ALICE Collaboration, Phys. Rev. Lett. 106, 032301 (2011). [arXiv, nucl-ex/1012,1657]

[3] The ALICE Collaboration, JHEP 1511, 127 (2015). [arXiv, nucl-ex/1506, 08808]

[4] A.G. Litvinenko, this volume

[5] M. Golubeva et al., Yad. Fiz. 76, 2 (2013)

[6] A. Ivashkin et al., MPD Forward Hadron Calorimeter (FHCAL), Technical Design Report, 1 (2016)

[7] S.G. Mashnik et al., arXiv:1607.02506 [nucl-th] 